\title{
DISFUNÇÃO SEXUAL EM MULHERES QUE FAZEM SEXO COM MULHERES
}

\author{
Lucas Feitosa de Souza', Daniela Siqueira Prado², Íkaro Daniel de Carvalho Barreto ${ }^{3}$ \\ SEXUAL DYSFUNCTION IN WOMEN WHO HAVE SEX WITH WOMEN \\ DISFRUNCIÓN SEXUAL EN MUJERES QUE HACEN SEXO CON MUJERES
}

\begin{abstract}
Resumo: $O$ objetivo do presente trabalho de pesquisa foi verificar se existe diferença na prevalência de disfunção sexual entre mulheres que fazem sexo com mulheres (MSM) e mulheres que fazem sexo apenas com homens (MSH), e se as percepções interna e externa do estigma acerca da orientação sexual se relacionam com a disfunção sexual. Metodologia: estudo transversal com 105 mulheres com idade superior a 18 anos e sexualmente ativas, sendo 35 do grupo MSM e 70 do grupo MSH. Avaliou-se aspectos sociodemográficos e aplicou-se o Índice de Função Sexual Feminina (IFSF) para todas as participantes e a Escala de Avaliação de Homofobia Internalizada apenas para mulheres que fazem sexo com mulheres. Para avaliar variáveis categóricas foram utilizados os testes Qui-Quadrado de Pearson, teste Exato de Fisher ou teste Qui-Quadrado de Pearson com simulações de Monte-Carlo. O nível de significância adotado foi de $5 \%$ e o software utilizado foi o R Core Team 2019. Resultados: a prevalência de disfunção sexual foi de 17,1\%. Não houve diferença significativa na média do escore do IFSF entre os grupos $(30,2 \pm 3,5 \times 29,8 \pm 3,3, p=0,5 I 8)$. Verificou-se melhor escore de desejo nas MSM $(4,8 \pm 0,9 \times 4,3 \pm 0,9, p=0,003)$. As percepções externa e interna do estigma acerca da orientação sexual não se relacionaram com a disfunção sexual. Conclusões: a prevalência de disfunção sexual foi relativamente baixa, não houve relação entre orientação sexual e disfunção sexual e as percepções interna e externa do estigma acerca da orientação sexual não se associaram à disfunção sexual.
\end{abstract}

Palavras-chave: Disfunção sexual. Orientação sexual. Homofobia internalizada.

Abstract: The objective of this research work was to verify if there is a difference in the prevalence of sexual dysfunction among women who have sex with women (WSW) and women who have sex with men only (WSM), and if the internal and external perceptions of sexual orientation stigma are related to sexual dysfunction. Methodology: a crosssectional study with 105 women over the age of 18 years and sexually active, being 35 of the group WSW and 70 of the group WSM. Sociodemographic aspects were evaluated and the Female Sexual Function Index (FSFI) was applied to all participants and the Internalized Homophobia Assessment Scale was applied only to women who have sex with women. Pearson's Chi-Square test, Fisher's exact test or Pearson's Chi-Square test with Monte-Carlo simulations were used to evaluate categorical variables. The significance level adopted was $5 \%$ and the R Core Team 2019 software was used. Results: the overall prevalence of sexual dysfunction was $17.1 \%$. There was no significant difference in the mean FSFI score between the groups $(30,2 \pm 3,5 \times 29,8 \pm 3,3, p=0.518)$. There was a better desire score in WSW $(4,8 \pm 0,9 \times 4,3 \pm 0,9$, $p=0.003)$. External and internal perceptions of stigma about sexual orientation were not related to sexual dysfunction. Conclusions: the prevalence of sexual dysfunction was relatively low, there was no relationship between sexual orientation and sexual dysfunction, and the internal and external perceptions of sexual orientation stigma were not associated with sexual dysfunction.

Keywords: Sexual dysfunction. Sexual orientation. Internalized homophobia.

Resumen: El objetivo de este trabajo de investigación era verificar si existe diferencia en la prevalencia de disfunción sexual entre mujeres que tienen sexo con mujeres (MSM) y mujeres que tienen sexo sólo con hombres (MSH), y si las percepciones interna y externa del estigma acerca de la orientación sexual se relacionan con la disfunción sexual. Me-

I Acadêmico do curso de Medicina da Universidade Federal de Sergipe (UFS), Aracaju (SE), Brasil.

2 Professora doutora do Curso de Medicina da Universidade Federal de Sergipe (UFS), Aracaju (SE), Brasil.

3 Doutorando no Programa de Pós-graduação em Biometria e Estatística Aplicada da Universidade Federal Rural de Pernambuco (UFRPE), Pernambuco, Brasil. 
todología: estudio transversal con 105 mujeres con edad superior a I 8 años y sexualmente activas, siendo 35 del grupo MSM y 70 del grupo MSH. Se evaluaron aspectos sociodemográficos y se aplicó el Índice de Función Sexual Femenina (IFSF) para todas las participantes y la Escala de Evaluación de Homofobia Internalizada sólo para mujeres que tienen sexo con mujeres. Para evaluar variables categóricas se utilizaron las pruebas Qui-cuadrado de Pearson, prueba Exacto de Fisher o prueba Qui-cuadrado de Pearson con simulaciones de Monte Carlo. El nivel de significancia adoptado fue del $5 \%$ y el software utilizado fue el R Core Team 2019. Resultados: la prevalencia global de disfunción sexual fue del 17,1\%. No hubo diferencia significativa en la media del score del IFSF entre los grupos $(30,2 \pm 3,5 \times 29,8 \pm 3,3, p=$ 0,5 I8). Se observó una mejor puntuación de deseo en las $M S M(4,8 \pm 0,9 \times 4,3 \pm 0,9, p=0,003)$. Las percepciones externa e interna del estigma acerca de la orientación sexual no se relacionaron con la dinsfunción sexual. Conclusiones: la prevalencia de disfunción sexual fue relativamente baja, no hubo relación entre orientación sexual y disfunción sexual y las percepciones interna y externa del estigma acerca de la orientación sexual no se asociaron a la disfunción sexual.

Palabras clave: Disfunción sexual. Orientación sexual. Homofobia internalizada.

\section{Introdução}

A disfunção sexual é definida, de acordo com a Associação Americana de Psiquiatria (APA) como transtorno que se caracteriza por uma perturbação, clinicamente significativa, na capacidade de experimentar prazer ou de ter uma resposta sexual adequada (AMERICAN PSYCHIATRIC ASSOCIATION, 20I4). A Disfunção Sexual Feminina (DSF) é uma condição que envolve determinantes biológicos, psicológicos e interpessoais (CEREJO, 2006).

O estudo epidemiológico americano National Health and Social Life Survey mostrou que $43 \%$ das mulheres americanas possuem algum tipo de disfunção sexual (LAUMANN; PAIK; ROSEN, 1999). Em estudo realizado na Índia, 72,3\% das mulheres participantes apresentaram disfunção sexual (SINGH et al., 2009). No Brasil, o Estudo do Comportamento Sexual detectou que $49 \%$ das mulheres possuíam algum tipo de disfunção sexual (ABDO et al., 2004). A DSF altera a qualidade de vida e das relações afetivas, sendo sua investigação recomendada pela Organização Mundial da Saúde (OMS) (CABRAL et al., 20I4; CHEDRAUl et al., 2008; SANTOS; LEÃO; GARDENGHI, 2016).

Existem vários fatores de risco que se relacionam com a disfunção sexual, entre eles doenças neurológicas, endócrinas ou vasculares, cirurgias genitais, antecedente de abuso sexual, nível educacional e socioeconômico, medicações como os anticoncepcionais, alterações hormonais fisiológicas como a menopausa e a amamentação, fatores psicológicos como a depressão e a ansiedade e fatores interpessoais como a má relação com o parceiro(a) e a discriminação por conta da orientação sexual (BERMAN; BERMAN; GOLDSTEIN, 1999; CEREJO, 2006; FAJEWONYOMI; ORJl; ADEYEMO, 2007; LIGHTNER, 2002; NAJAFABADY; SALMANI; ABEDI, 20II; PABLO; SOARES, 2004).
As mulheres com práticas homoafetivas e homoeróticas relatam experiências de discriminação por familiares ou por amigos/vizinhos, além de não acessarem com frequência serviços de saúde. Os medos relacionados ao preconceito, seus efeitos na vida cotidiana e nas relações sociais, a discriminação e o mistério que estão associados às relações afetivo-sexuais entre mulheres, contribuem para o silêncio e restrições que podem implicar negativamente nas relações afetivas e sexuais (RODRIGUES, 2010).

Para Koehler (20I3) a homofobia é conceituada como rejeição, aversão, medo ou ódio irracional aos homossexuais, consequentemente, todos aqueles que demonstram orientação sexual ou identidade de gênero diferente dos padrões heterossexuais ainda aceitos como normativos na nossa sociedade, sofrem com o comportamento homofóbico.

Nesse contexto, a homofobia internalizada, comumente definida como medo da própria homossexualidade, é um fenômeno cultural, que não é universal, nem toma as mesmas formas ou o mesmo significado em diferentes grupos sociais, comunidades e regiões. $\mathrm{O}$ conceito de homofobia internalizada tem sido apresentado por muitos autores como um fator central na manifestação de vários aspectos relacionados com saúde e doença entre pessoas homossexuais (WEBER-GILMORE; ROSE; RUBENSTEIN, 20I2). A ansiedade, a vergonha e a desvalorização do eu LGB são inerentes à homofobia internalizada e tendem a ser abertamente manifestadas nas relações interpessoais. Ao experimentar esses sentimentos negativos na composição interpessoal íntima, a relação torna-se suscetível a uma menor qualidade e satisfação, em especial na esfera sexual (PEREIRA; LEAL, 2002).

Tendo em vista o fato da orientação sexual e da homofobia internalizada associarem-se significativamente com as relações interpessoais, no contexto afetivo e sexual, este estudo tem como objetivo verificar se existe diferença na prevalência de disfunção sexual entre mulheres 
que fazem sexo com mulheres (MSM) e mulheres que fazem sexo apenas com homens (MSH) e se as percepções interna e externa do estigma acerca da orientação sexual se relacionam com a disfunção sexual.

\section{Metodologia}

Trata-se de estudo do tipo transversal, realizado no período entre outubro de 2018 e janeiro de 2019, através da aplicação de questionários em palestras realizadas no Hospital Universitário da Universidade Federal de Sergipe (UFS), assim como nas aulas da Liga Acadêmica de Saúde LGBT de Sergipe da UFS. Um total de 105 mulheres participaram do estudo, sendo divididas em dois grupos: um de MSM, composto por 35 participantes e outro de MSH, composto por 70 participantes.

Foram incluídas na pesquisa, mulheres cisgênero que concordaram em participar por meio da assinatura do Termo de Consentimento Livre e Esclarecido (TCLE); que tinham 18 anos ou mais na ocasião da entrevista; residiam no estado de Sergipe há pelo menos seis meses; e que tinham vida sexual ativa, que nesse estudo foi definida como ter tido relação sexual nas últimas quatro semanas.

Foram excluídas da pesquisa mulheres que se recusaram a assinar o TCLE; mulheres com estados fisiológicos que pudessem interferir na função sexual como: gestantes, puérperas e as com status pós-menopausa.

Inicialmente foram avaliados aspectos sociodemográficos como: idade, nível de escolaridade, parceria (fixa ou variável), se teve relações sexuais nas últimas quatro semanas e a orientação sexual. Estratificou-se o nível de escolaridade em I a 3 anos de estudo, 4 a 7 anos de estudo, 8 anos ou mais de estudo e não determinado. As mulheres puderam classificar a sua orientação sexual em heterossexual, homossexual, bissexual ou outra.

A seguir, aplicou-se o Índice de Função Sexual Feminina (IFSF) tanto para o grupo MSM quanto para o grupo MSH. O IFSF é uma escala breve, específica e multidimensional, que apresenta significante confiabilidade e validade (LEITE et al., 2007), e transforma medidas subjetivas em dados objetivos, quantificáveis e analisáveis. O ISFH foi constituído de 19 perguntas agrupadas em seis domínios: desejo sexual, excitação sexual, lubrificação vaginal, orgasmo, satisfação sexual e dor ou desconforto. Cada resposta do IFSF recebe uma pontuação entre 0 e 5 . Para calcular o escore total de cada domínio, deve-se contabilizar a soma dos valores das questões, multiplicar essa soma pelo fator de correção e depois somar os valores de cada domínio. Os escores finais podem variar de 2 a 36 , sendo que valores mais altos indicam um grau melhor de função sexual, mulheres com escores $\leq 26,55$ devem ser consideradas portadoras de disfunção sexual (ROSEN et al., 2000).

Posteriormente, foi aplicada a Escala de Avaliação de Homofobia Internalizada, apenas para o grupo MSM.
Essa escala consiste de 26 itens, sendo uma escala do tipo Likert, variando de I - "discordo totalmente" a 7 - "concordo totalmente". Na adaptação portuguesa da escala, o termo homossexual é utilizado para lésbicas, gays e bissexuais, sendo também adaptadas às questões que se referiam especificamente à homossexualidade masculina para a homossexualidade feminina (e.g. "Homens homossexuais obviamente afeminados fazem-me sentir desconfortável." para "Mulheres homossexuais obviamente masculinas fazem-me sentir desconfortável." (CARREIRAS, 2014). Tal escala é capaz de classificar a homofobia internalizada em duas dimensões, sendo elas a percepção interna do estigma acerca da homossexualidade e a percepção externa do estigma acerca da homossexualidade (PEREIRA; LEAL, 2005). Os itens 3, I3, I5, I7, I8, 20 e 24 dizem respeito à percepção externa do estigma associado à homossexualidade, e os demais itens estão relacionados a percepção interna do estigma associado à homossexualidade. Uma vez que a escala utiliza uma forma de cotação de Likert de I a 7, o valor médio é de 3,5, após a correção dos itens inversos, o que significa que $M>3,5$ revela níveis elevados de percepção interna e externa do estigma acerca da homossexualidade e, quando $M<3,5$ revela baixa percepção interna e externa do estigma acerca da homossexualidade.

As variáveis categóricas foram descritas por meio de frequência absoluta e relativa percentual. As variáveis contínuas foram descritas por meio de média, mediana, desvio padrão e intervalo interquartil. As associações foram testadas por meio de teste Qui-Quadrado de Pearson, teste Exato de Fisher ou teste Qui-Quadrado de Pearson com simulações de Monte-Carlo. A aderência à distribuição normal das variáveis contínuas foi testada por meio do teste de Shapiro-Wilks. As diferenças de medida de tendência central foram testadas por meio do teste $T$ para amostras independentes quando atendem o pressuposto de normalidade e por meio do teste de Mann-Whitney quando não atendem. O nível de significância adotado foi de $5 \%$ e o software utilizado foi o R Core Team 2019.

Este estudo foi apreciado e aprovado pelo Comitê de Ética em Pesquisa em Seres Humanos da Universidade Federal de Sergipe (CAAE 82525917.8.0000.5546).

\section{Resultados}

A média de idade das participantes foi de 24,5 $( \pm 5,5)$. A maioria das mulheres não possuíam parceria sexual fixa $(76,2 \%)$ e tinham oito anos de estudo ou mais (91,4\%). Quanto à orientação sexual, as mulheres se distribuíram da seguinte maneira: heterossexual $(66,7 \%)$, homossexual $(15,2 \%)$, bissexual $(16,2 \%)$ e panssexual $(\mathrm{I}, 9 \%)$ (Tabela I).

A prevalência global de disfunção sexual (IFSF $\leq$ 26,55 ) foi de $17,1 \%$ e a média geral do escore foi de 29,9 
$( \pm 3,4)$. Quanto à associação entre disfunção sexual e aspectos sociodemográficos, verificou-se que as mulheres com parceria fixa apresentaram maior frequência de disfunção sexual que aquelas sem parceria fixa $(36,0 \%$ $x \mid I, 2 \%, p=0,012)$. Relação entre o nível de escolaridade e disfunção sexual não foi observada nesse estudo $(p=0$, I I5) (Tabela 2).

Não houve diferença na prevalência de DSF por grupo (MSM 17,1\% x MSH 17,1\%, p=I,000) (Tabela 2). Em relação à avaliação das médias do escore do IFSF, também não houve diferença entre os grupos (MSM $30,2 \pm 3,5 \times \mathrm{MSH} 29,8 \pm 3,3, \mathrm{p}=0,518$ ). Ao comparar os escores dos domínios da função sexual entre os dois grupos, percebeu-se melhor escore de desejo no grupo $\operatorname{MSM}(4,8 \pm 0,9 \times 4,3 \pm 0,9, p=0,003)$, enquanto nos outros domínios não houve diferença significativa (Tabela 3).

Quanto à percepção do estigma acerca da orientação sexual, verificada pela Escala de Homofobia Internalizada, o grupo MSM apresentou valores elevados para o escore de percepção externa do estigma $(5, I \pm 0,9)$ e baixos para o escore de percepção interna do estigma $(2,2 \pm 0,6)$. Ao correlacionar os escores da percepção do estigma acerca da orientação sexual com a presença de disfunção sexual no grupo MSM, verificou-se que não houve associação entre eles, tanto em relação à percepção externa do estigma $(p=0,66 I)$ quanto em relação a percepção interna do estigma $(p=0,166)$ (Tabela 4).

\section{Discussão}

A disfunção sexual tem etiologia multifatorial e complexa, podendo possuir causas orgânicas ou psicogênicas. Mulheres que sofrem de depressão e ansiedade podem apresentar maior frequência de disfunção sexual (COLSON et al., 2006). As MSM são vítimas de discriminação e preconceitos, que podem levar a distúrbios psíquicos que impactem negativamente em sua função sexual.

A prevalência global de disfunção sexual do presente estudo $(17,1 \%)$ foi semelhante à encontrada em estudo composto por 244 mulheres, realizado em Sergipe em 2012, que mostrou uma prevalência de $17,6 \%$ de disfunção sexual (PRADO et al., 2014). Outro estudo, com 201 mulheres de níveis socioeconômicos distintos, também realizado em Sergipe em 2009, apresentou similaridade quanto à prevalência global (21,9\%) (PRADO; PAULA; LIMA, 20I0). Revisão sistemática, que avaliou a prevalência de DSF nas diferentes regiões do Brasil, mostrou prevalências entre 3I,2\% e 79,3\% na região Nordeste, superior à encontrada neste estudo (WOLPE et al., 2017). A pesquisa brasileira Brazilian Study on Sexual Behavior (BSSB), com I.219 mulheres, apresentou uma prevalência de 49\% (ABDO et al., 2004), assim como em estudo epidemiológico americano, que observou que $43 \%$ das mulheres americanas possuíam algum tipo de disfunção sexual (LAUMANN; PAIK; ROSEN, 1999). Destaca-se que dados referentes à prevalência de disfunção sexual, geralmente, possuem uma grande variação entre si, provavelmente por existirem diversos sistemas de avaliação, métodos de classificação e grupos populacionais presentes nesses estudos (BEAN, 2002), assim como o fato de muitas pesquisas serem realizadas de forma inadequada (NAZARETH; BOYNTON; KING, 2003). A média de idade das pacientes do presente estudo foi baixa, o que pode explicar a baixa frequência de disfunção sexual.

Quanto aos dados sociodemográficos, não foi verificada associação entre nível de escolaridade e disfunção sexual, provavelmente pelo fato de o grupo ter sido, em sua maior parte, composto por mulheres com oito anos ou mais de estudo (91,4\%). Existem estudos que mostram uma relação entre maior nível de escolaridade e menor disfunção sexual (ABDO et al., 2004; SHIFREN et al., 2008), o que também pode justificar a baixa frequência de disfunção sexual deste estudo. Em relação ao tipo de parceria, percebeu-se associação positiva entre disfunção sexual e parceria fixa, diferente do demonstrado em estudo realizado no Ceará, que verificou maior prevalência em mulheres sem parceria fixa (BEZERRA et al., 2018). Estudo realizado por Pereira, Nardi e Silva (2013) mostrou uma prevalência maior de ansiedade em mulheres casadas em relação às não casadas, o que pode explicar a presença de maior prevalência de disfunção sexual nestas mulheres.

Não houve diferença na prevalência de disfunção sexual nos grupos MSM e MSH, semelhante a estudos que apresentaram prevalências equivalentes ou inferiores em MSM quando comparadas a MSH (BREYER et al., 20I0; LAUMANN; PAIK; ROSEN, 1999; NICHOLS, 2004). No entanto, estudo britânico verificou maior prevalência de disfunção sexual no grupo MSM (23\%) que no MSH (19\%) (BURRI et al., 20I2). Em relação à comparação dos escores por domínios, percebeu-se melhor escore de desejo no grupo MSM, ao contrário do apresentando pelo estudo de Burri e outros (2012) que mostrou escores piores de desejo e satisfação no grupo MSM. Nos demais domínios os grupos não divergiram significativamente, diferente do exposto no estudo de Nichols (2004) em que as MSM apresentaram menor dificuldade de atingir $o$ orgasmo $(p<0,03)$, menos problemas com a lubrificação $(p<0,003)$ e menos dor durante a penetração vaginal $(p<0,005)$.

Em relação à percepção do estigma acerca da orientação sexual, todas as MSM apresentaram valores elevados para o escore de percepção externa do estigma e baixos para o escore de percepção interna do estigma, sem relação com a frequência de disfunção sexual. Essa falta de correlação pode ser explicada, talvez, pelo fato do 
grupo se comportar de forma homogênea em relação à percepção do estigma.

Este estudo abordou tema pouco explorado na literatura no que diz respeito à saúde sexual de mulheres que fazem sexo com mulheres. Contudo, é importante destacar como limitação, o fato das mulheres que constituíram a amostra participarem ativamente de grupos de discussões sobre saúde da população LGBT, acompanhando as aulas da Liga Acadêmica de Saúde LGBT de Sergipe, possuindo assim mais instrução acerca do tema e, consequentemente, apresentarem-se mais bem resolvidas quanto à sua orientação sexual.

\section{Conclusão}

Pode-se concluir que a prevalência de disfunção sexual foi relativamente baixa, que não houve relação entre orientação sexual e disfunção sexual e que a percepção interna e externa do estigma acerca da orientação sexual não se associou com disfunção sexual.

A grande variação da prevalência de DSF entre os valores encontrados nesta pesquisa e os valores de diferentes países e diferentes regiões de um mesmo país, pode ser justificada, também, pelas interferências de aspectos culturais e sociais incorporadas pelas pessoas, atitudes e valores nas construções das identidades que diferem do modelo heteronormativo. Considerando essa variação, surge a necessidade de realização de novas pesquisas para que se possam confrontar os resultados que foram encontrados em diferentes localidades.

Tabela I - Caracterização sociodemográfica da população segundo a orientação sexual

\begin{tabular}{|c|c|c|c|}
\hline & \multicolumn{2}{|c|}{ Orientação sexual } & \multirow[b]{2}{*}{ p-valor } \\
\hline & $\begin{array}{l}\text { MSM } \\
\text { N (\%) }\end{array}$ & $\begin{array}{c}\text { MSH } \\
\text { N (\%) }\end{array}$ & \\
\hline \multicolumn{4}{|l|}{ Parceria } \\
\hline Com parceria fixa & II $(3 \mathrm{I}, 4)$ & $14(20)$ & $0,228^{F}$ \\
\hline Sem parceria fixa (variável) & $24(68,6)$ & $56(80)$ & \\
\hline \multicolumn{4}{|l|}{ Nível de escolaridade } \\
\hline I a 3 anos de estudo & I $(2,9)$ & I $(I, 4)$ & I,000 QM \\
\hline 4 a 7 anos de estudo & I $(2,9)$ & $3(4,3)$ & \\
\hline 8 anos de estudo ou mais & $32(91,4)$ & $64(91,4)$ & \\
\hline Não determinada & I $(2,9)$ & $2(2,9)$ & \\
\hline
\end{tabular}

Legenda: $\mathrm{N}$ - frequência absoluta. \% - frequência relativa percentual. ${ }^{\mathrm{F}}$ Teste Exato de Fisher. ${ }^{\mathrm{OM}}$ Teste Qui-Quadrado de Pearson com simulações de Monte-Carlo.

Fonte: elaborada pelos autores.

Tabela 2 - Correlação entre orientação sexual e aspectos sociodemográficos com o Índice de Função Sexual Feminina (IFSF) (continua)

\begin{tabular}{|c|c|c|c|c|c|}
\hline & & & \multicolumn{2}{|c|}{ IFSF total } & \multirow[b]{2}{*}{ p-valor } \\
\hline & $\mathrm{N}$ & $\%$ & $\begin{array}{c}\leq 26,55 \\
N(\%)\end{array}$ & $\begin{array}{c}>26,55 \\
N(\%)\end{array}$ & \\
\hline \multicolumn{6}{|c|}{ Orientação sexual } \\
\hline $\mathrm{MSH}$ & 70 & 66,7 & $12(\mid 7, I)$ & $58(82,9)$ & $\mathrm{I}, 000^{\mathrm{F}}$ \\
\hline
\end{tabular}




\begin{tabular}{|c|c|c|c|c|c|}
\hline & & & \multicolumn{2}{|c|}{ IFSF total } & \multirow[b]{2}{*}{ p-valor } \\
\hline & $\mathrm{N}$ & $\%$ & $\begin{array}{c}\leq 26,55 \\
N(\%)\end{array}$ & $\begin{array}{c}>26,55 \\
N(\%)\end{array}$ & \\
\hline MSM & 35 & 33,3 & $6(17, I)$ & $29(82,9)$ & \\
\hline \multicolumn{6}{|l|}{ Parceria } \\
\hline Com parceria fixa & 25 & 23,8 & $9(36,0)$ & $16(64,0)$ & $0,012^{\mathrm{F}}$ \\
\hline Sem parceria fixa (variável) & 80 & 76,2 & $9(11,2)$ & $71(88,8)$ & \\
\hline \multicolumn{6}{|l|}{ Nível de escolaridade } \\
\hline I a 3 anos de estudo & 2 & 1,9 & $0(0)$ & $2(100,0)$ & $0,115 \mathrm{Qm}$ \\
\hline 4 a 7 anos de estudo & 4 & 3,8 & I $(25,0)$ & $3(75,0)$ & \\
\hline 8 anos de estudo ou mais & 96 & 91,4 & $15(15,6)$ & $8 \mathrm{I}(84,4)$ & \\
\hline Não determinada & 3 & 2,9 & $2(66,7)$ & I $(33,3)$ & \\
\hline
\end{tabular}

Legenda: $\mathrm{N}$ - frequência absoluta. \% - frequência relativa percentual. ${ }^{\mathrm{F}}$ Teste Exato de Fisher. ${ }^{\mathrm{QM}}$ Teste Qui-Quadrado de Pearson com simulações de Monte-Carlo.

Fonte: elaborada pelos autores.

Tabela 3 - Média do Índice de Função Sexual Feminina (IFSF) e dos domínios da função sexual segundo a orientação sexual

\begin{tabular}{|c|c|c|c|c|}
\hline & \multirow[b]{2}{*}{$\begin{array}{c}\text { Geral } \\
\text { Média (DP) }\end{array}$} & \multicolumn{2}{|c|}{ Orientação sexual } & \multirow[b]{2}{*}{ p-valor } \\
\hline & & $\begin{array}{c}\text { MSM } \\
\text { Média (DP) }\end{array}$ & $\begin{array}{c}\text { MSH } \\
\text { Média (DP) }\end{array}$ & \\
\hline Idade & $24,5(5,5)$ & $24,2(4)$ & $24,6(6,2)$ & $0,538 w$ \\
\hline IFSF & $29,9(3,4)$ & $30,2(3,5)$ & $29,8(3,3)$ & $0,518^{\top}$ \\
\hline Desejo & $4,5(I)$ & $4,8(0,9)$ & $4,3(0,9)$ & $0,003 w$ \\
\hline Excitação & $5(0,8)$ & $5(0,7)$ & $4,9(0,8)$ & $0,986 \mathrm{w}$ \\
\hline Lubrificação & $5, I(0,8)$ & $5,3(0,6)$ & $5, I(0,9)$ & $0,40 I^{w}$ \\
\hline Orgasmo & $5(0,8)$ & $5, I(0,7)$ & $4,9(0,9)$ & $0,321 \mathrm{w}$ \\
\hline Satisfação & $5,4(0,8)$ & $5,3(0,8)$ & $5,5(0,8)$ & $0,202 w$ \\
\hline Dor & $5(1,3)$ & 4,8 $(I, 8)$ & $5,1(1)$ & $\mathrm{I}, 000 \mathrm{w}$ \\
\hline
\end{tabular}

Legenda: DP - Desvio Padrão. ${ }^{\top}$ Teste T para amostras independentes. ${ }^{\mathrm{w}}$ Teste de Mann-Whitney.

Fonte: elaborada pelos autores. 
Tabela 4 - Correlação entre o score da Escala de Avaliação da Homofobia Internalizada (dividído nas dimensões: percepção externa e percepção interna) e do Índice de Função Sexual Feminina (IFSF)

\begin{tabular}{|l|c|c|c|c|}
\hline \multirow{2}{*}{} & \multicolumn{2}{|c|}{ ISFS } \\
\cline { 2 - 5 } & $\begin{array}{c}\text { Geral } \\
\text { Média (DP) }\end{array}$ & $\begin{array}{c}\leq 26,55 \\
\text { Média (DP) }\end{array}$ & $\begin{array}{c}>26,55 \\
\text { Média (DP) }\end{array}$ & P-valor \\
\hline Escore Percepção Externa & $5, I(0,9)$ & $4,9(0,4)$ & $5, I(0,9)$ & $0,66 I^{\top}$ \\
\hline Escore Percepção Interna & $2,2(0,6)$ & $2,5(0,5)$ & $2, I(0,6)$ & $0, I^{\top} 66^{\top}$ \\
\hline
\end{tabular}

Legenda: DP - Desvio Padrão. ${ }^{\top}$ Teste T para amostras independentes.

Fonte: elaborada pelos autores.

\section{Referências}

ABDO, C. et al. Prevalence of sexual dysfunctions and correlated conditions in a sample of Brazilian women - results of the Brazilian study on sexual behavior (BSSB). International Journal of Impotence Research, v. I6, p. I60-166, 2004.

AMERICAN PSYCHIATRIC ASSOCIATION. DSM-5: Manual diagnóstico e estatístico de transtornos mentais. 5. ed. Porto Alegre: Artmed Editora, 2014.

BEAN, J. L. Expressions of Female Sexuality. Journal of Sex and Marital Therapy, v. 28, p. 29-38, 2002.

BERMAN, J. R.; BERMAN, L.; GOLDSTEIN, I. Female sexual dysfunction: incidence, pathophysiology, evaluation, and treatment options. Urology, v. 54, n. 3, p. 385-39I, 1999.

BEZERRA, K. D. C. et al. Função sexual de universitárias: estudo comparativo entre Brasil e Itália. Revista Brasileira de Enfermagem, v. 7I, suppl 3, p. I5II-I5I7, 2018.

BREYER, B. N. et al. The Impact of Sexual Orientation on Sexuality and Sexual Practices in North American Medical Students. Journal of Sexual Medicine, v. 7, p. 239I-2400, 2010.

BURRI, A. et al. The relationship between samesex sexual experience, sexual distress, and female sexual dysfunction. Journal of Sexual Medicine, v. 9, n. I, p. 198-206, 2012.
CABRAL, P. U. L. et al. Physical activity and sexual function in middle-aged women. Revista da Associação Médica Brasileira, v. 60, n. I, p. 47-52, 2014.

CARREIRAS, L. M. C. Autoestima sexual, identidade e homofobia internalizada numa população LGB. Faro: Universidade do Algarve, 2014.

CEREJO, A. C. Disfunção sexual feminina: prevalência e factores relacionados. Revista Portuguesa de Medicina Geral e Familiar, v. 22, p. 70I-720, 2006.

CHEDRAUI, P. et al. Impaired quality of life among middle aged women: A multicentre Latin American study. Maturitas, v. 6I, p. 323-329, 2008.

COLSON, M. H. et al. Sexual behaviors and mental perception, satisfaction and expectations of sex life in men and women in France. Journal of Sexual Medicine, v. 3, n. I, p. 12।-13।, 2006.

FAJEWONYOMI, B. A.; ORJI, E. O.; ADEYEMO, A. O. Sexual dysfunction among female patients of reproductive age in a hospital setting in Nigeria. Journal of Health, Population and Nutrition, v. 25, n. I, p. I0I-106, 2007.

KOEHLER, S. M. F. Homofobia, cultura e violências: a desinformação social. Interacções, v. 26, p. $|29-| 5|, 20| 3$.

LAUMANN, E. O.; PAIK, A.; ROSEN, R. C. Sexual Dysfunction in the United States. JAMA, v. 28I, n. 6, p. 537-544, 1999. 
LEITE, A. P. L. et al. Validation of the female sexual function index in Brazilian pregnant women. Revista Brasileira de Ginecologia e Obstetrícia, v. 29, n. 8, p. 4I4-4I9, 2007.

LIGHTNER, D. J. Female Sexual Dysfunction. Mayo Clinic Proceedings, v. 77, p. 698-702, 2002.

NAJAFABADY, M. T.; SALMANI, Z.; ABEDI, P. Prevalence and related factors for anorgasmia among reproductive aged women in Hesarak, Iran. Clinics, v. 66, n. I, p. 83-86, 20 I I.

NAZARETH, I.; BOYNTON, P.; KING, M. Problems with sexual function in people attending London general practitioners: cross sectional study. British Medical Journal, v. 327, p. I-6, 2003.

NICHOLS, M. Lesbian sexuality/female sexuality: Rethinking "lesbian bed death". Sexual and Relationship Therapy, v. 19, n. 4, p. 363-37I, 2004.

PABLO, C.; SOARES, C. As disfunções sexuais femininas. Revista Portuguesa de Clínica Geral, v. 20, p. 357-369, 2004.

PEREIRA, H.; LEAL, I. P. A homofobia internalizada e os comportamentos para a saúde numa amostra de homens homossexuais. Análise Psicológica, v. I, n. XX, p. 107-II3, 2002.

PEREIRA, H.; LEAL, I. P. Medindo a homofobia internalizada: a validação de um instrumento. Análise Psicológica, v. 3, n. XXIII, p. 323-328, 2005.

PEREIRA, V. M.; NARDI, A. E.; SILVA, A. C. Sexual dysfunction, depression, and anxiety in young women according to relationship status: an online survey. Trends in Psychiatry and Psychotherapy, v. 35, n. I, p. 55-6I, 2013.

PRADO, D. S. et al. Impacto da infecção por HIV e da terapia antirretroviral sobre a função sexual feminina. Revista Brasileira de Sexualidade Humana, v. 26, n. I, p. 19-25, 2014.

PRADO, D. S.; PAULA, V. L. P. M.; LIMA, T. I. A. Prevalência de disfunção sexual em dois grupos de mulheres de diferentes níveis socioeconômicos. Revista Brasileira de Ginecologia e Obstetrícia, v. 32, n. 3, p. I39-I43, 2010.

RODRIGUES, J. L. Saúde sexual e reprodutiva de mulheres lésbicas e bissexuais. Fazendo gênero 9, p. I-II, 2010.
ROSEN, R. et al. The Female Sexual Function Index (FSFI): A Multidimensional Self-Report Instrument for the Assessment of Female Sexual Function. Journal of Sex and Marital Therapy, v. 26, n. 2, p. |9|-208, 2000.

SANTOS, J. DE L.; LEÃO, A. P. F.; GARDENGHI, G. Disfunções sexuais no climatério. Reprodução e Climatério, v. 31, n. 2, p. 86-92, 2016.

SHIFREN, J. L. et al. Sexual Problems and Distress in United States Women. Obstetrics and Gynecology, v. II 2, n. 5, p. 970-978, 2008.

SINGH, J. et al. Prevalence and risk factors for female sexual dysfunction in women attending a medical clinic in south India. Journal of Postgraduate Medicine, v. 55, n. 2, p. II 3, 2009.

WEBER-GILMORE, G.; ROSE, S.; RUBENSTEIN, R. The Impact of Internalized Homophobia on Outness for Lesbian, Gay, and Bisexual Individuals. The Professional Counselor, v. I, n. 3, p. I63-I75, 2012.

WOLPE, R. E. et al. Prevalence of female sexual dysfunction in Brazil: A systematic review. European Journal of Obstetrics Gynecology and Reproductive Biology, v. 2II, p. 26-32, 2017. 